\title{
INDEX OF NAMES OF SYNTAXA TYPIFIED IN 1994
}

\author{
Jean-Paul Theurillat ${ }^{1)}$ \& Jaroslav Moravec ${ }^{2)}$ \\ 1) Centre alpien de Phytogéographie, Fondation J.-M. Aubert, CH-1938 Champex, and Conservatoire et \\ Jardin botaniques, Case postale 60, CH-1292 Chambésy, Switzerland; fax +41224185101 , \\ E-mail theurillat@cjb.unige.ch \\ 2) Institute of Botany, Academy of Sciences of the Czech Republic, CZ-252 43 Prühonice, Czech Republic; \\ $f a x+420267750031$
}

Keywords: Alliances, Associations, Classes, Code of Phytosociological Nomenclature, Orders, Phytosociology, Typification

\begin{abstract}
Following the "Index of new names" (THEURILLAT \& MORAVEC 1998), the present work collects the names of syntaxa (in the sense of the Code of phytosociological nomenclature, BARKMAN et al. 1986) above subassociation rank typified in 1994. The list comprises 101 names of syntaxa; three names are added to the Index 1992 (THEURILLAT \& MORAVEC 1995).
\end{abstract}

The "Index 1994" contains 101 typifications of names of syntaxa higher than the subassociation rank (sensu BARKMAN et al. 1986). In the addendum three typifications are added to the "Index 1992" (THEURILLAT \& MORAVEC 1995). These names were compiled following consultation of the literature at the library of the Conservatoire et Jardin botaniques of the city of Geneva. The "Index" follows the same principles as "Index 1987" (THEURILLAT \& MORAVEC 1990) to which reference will be made.

The symbols $(O, *, \Delta)$ preceding the names indicate the nomenclatural legitimacy of the typification. Illegitimate or superfluous typifications - referring to articles 16-21 of the Code of phytosociological nomenclature - are preceded by $O$, i.e. 12 cases $(12 \%)$ for the present "Index". The asterisk (*) indicates a typification requiring further bibliographical or nomenclatural verifications of its validity or authority ( 2 for the present "Index"). The category of "unchecked names" $(\Delta)$, introduced with the "Index 1993", includes for verification those names whose validity could not be readily verified because the required bibliographical references were not available to the authors. In this way they are removed from the category of doubtful typifications in which they were previously included. The present "Index" contains 42 unchecked names (i.e. $41 \%$ ).

With its 104 typifications, the following list may not be complete and the authors would welcome offprints concerning missing elements. Observations concerning the validity of typifications requiring further verification as well as of unchecked typifications would be also appreciated.

\section{INDEX}

Communities of cryptogams

Cystoseiretea

$\Delta$ Cystoseirion MOLINIER 1958. In: GIACCONE et al. 1994 [1995?] (: 130) 
$\Delta$ Sagassion hornschuchii GIACCONE 1973. In: GIACCONE et al. 1994 [1995?] (: 130)

$\Delta$ Chaetomorpho-Valonietum aegagropilae GIACCONE 1974. In: GIACCONE et al. 1994 [1995?] (: 136)

$\Delta$ Cystoseiretum barbatae PIGNATTI 1962. In: GiacConE et al. 1994 [1995?] (: 121)

$\triangle$ Cystoseiretum crinitae MOLINIER 1958. In: GIACCONE et al. 1994 [1995?] (: 118)

$\Delta$ Cystoseiretum dubiae Furnari, SCAMmaCCA, CORMaCi et Battiato 1977. In: GiacCONE et al, 1994 [1995?] (: 128)

$\Delta$ Cystoseiretum strictae MOLINIER 1958. In: GIACCONE et al. 1994 [1995?] (: 115)

$\Delta$ Cystoseiretum spinosae GIACCONE 1973. In: GIACCONE et al.1994 [1995?] (: 124)

$\Delta$ Cystoseiretum usneoidis GIACCONE 1972. In: GIACCONE et al. 1994 [1995?] (: 127)

$\Delta$ Cystoseiretum zosteroidis GiACCONE 1973. In: GIACCONE et al. 1994 [1995?] (: 126)

$\Delta$ Dasycladetum vermicularis MAYHOUB 1976. In: GlACCONE et al. 1994 [1995?] (: 117)

$\Delta$ Gracilarietum verrucosae GIACCONE 1974. In: GIACCONE et al. 1994 [1995?] (: 136)

$\triangle$ Myrionemo-Giraudietum sphacelarioidis VAN DER BEN 1991. In: GIACCONE et al. 1994 [ 1995 ?] (: 132)

$\triangle$ Sargassetum vulgaris MAYHOUB 1976. In: GIACCONE et al. 1.994 [1995?] (: 117)

$\Delta$ Trichosoletum myurae MAYHOUD 1976. In: GIACCONE et al. 1994 [1995?] (: 121)

\section{Communities of vascular plants}

Asplenietea trichomanis

Globularienion incanescentis BARBERO et BONO 1973. In: TOMASELLI 1994 (: 40)

$\triangle$ Anogrammo-Davallietum canariensis BELlot et CASASECA in CASASECA 1959. In: DÍAZ GONZÁLEZ \& FERNÁNDEZ PRIETO 1994 (: 438)

Valeriano-Saxifragetum BARBERO et BONO 1973. In: TOMASELli 1994 (: 40)

Cakiletea maritimae

$\Delta$ Atriplicetum hastato-tornabeni BOLòs 1962. In: GÉHU \& BIONDI 1994 (: 141)

Calluno-Ulicetea

Gentiano pneumonanthes-Ericetum mackaianae TÜXEN et OBERDORFER 1958. In: DÍAZ GONZÁL.EZ \& FERNÁNDEZ PRIETO 1994 (: 394)

Ulici-Ericetum GUINEA 1949. In: DÍAZ GONZÁLEZ \& FERNÁNDEZ PRIETO 1994 (: 392)

Crithmo-Limonietea

O Crithmo-Staticetum articulatae R. et R. MOLINIER 1955. In: GÉHU \& BIONDI 1994 typific. illeg. (: 77) [art. 19] [sub: Crithmo maritimi-Limonietum contortiramei (R. et R. MOLINIER 1955) GÉHU et BIONDI 1994 nom. nov.]

Elyno-Seslerietea

Caricion austroalpinae SUTTER 1962. In: FEOLI CHIAPELLA \& POLDINI 1994 (: 16)

Avenastro parlatorei-Festucetum calvae AICHINGER 1933 corr. FRANZ 1980. In: FEOLI CHIAPELLA \& POLDINI 1994 $(: 72)$

Euphorbio-Ammophiletea

O Sileno corsicae-Agrapyretum juncei Bartolo, Brullo, De MarCo, Dinelli, Signorello et SPAMPINATO 1992. In: GÉHU \& BIONDI 1994 typific. illeg. et superfl. (: 140) [art. 16, 19]

[sub: Sileno corsicae-Elymetum farcti (MALCUIT 1926) BARTOLO et al. 1992]

Festuco-Brometea

O Festuco-Brometea Braun-Blanquet et TÜXEN ex KLIKa et HadaČ 1944 nom. inval. In: Dengl.ER 1994 typific. superfl. (: 241$)$

$\Delta$ Hypochoeridion maculatae HoRvatić 1973. In: FEOLI ChIAPELla \& POLDINI 1994 (: 12)

$\Delta$ Saturejion subspicatae (HoRvat 1962) HoRvatić 1973. In: FEOLI ChIAPELla \& POLdiNi 1994 (: 11 )

$\Delta$ Scorzonerion villosae HoRvatić 1963. In: FEOLi ChIAPElla \& POLDINI 1994 (: 12)

Adonido-Brachypodietum pinnati (LiBBERT 1933) KRAUSCH 1961. In: DENGLER 1994 (: 263) 
Centaureo dichroanthae-Globularietum cordifoliae PIGNATTI 1953. In: FEOLI ChIAPELLA \& POLDINI 1994 (: 17) [neotypus]

$\Delta$ Potentillo arenariae-Stipetum capillatae (HUECK 1931) LIBBERT 1933. In: DENGLER 1994 (: 257)

Helichryso-Crucianelietea maritimae

Crucianello-Armerietum pungentis ZEVACO 1969. In: GÉHU \& BIONDI 1994 (: 30)

Thymelaeo hirsutae-Helichrysetum italici MOLINIER 1959. In: GÉHU \& BIONDI 1994 (: 100)

Honckenyo-Elymetea

$\triangle$ Tournefortietum sibiricae POPESCU et SANDA 1975. In: DU8YNA et al. 1995 (: 201)

Koelerio-Corynephoretea

$\Delta$ Festuco psammophilae-Koelerietum glaucae KLIKA 1931. In: DENGLER 1994 (: 241) Sileno otitae-Festucetum LIBBERT 1933. In: DENGLER 1994 (: 250)

Lem nete a

Lemnetum trisulcae DEN HaRTOG et SEGAL 1964. In: WOLFF et al. 1994 (: 350$)$

Lemno-Spirodeletum polyrmizae KoCH 1954. In: WOLFF et al. 1994 (: 349)

$\Delta$ Spirodelo polyrrhizae-Salvinietum natantis SLAVNIĆ 1956. In: WOLFF et al. 1994 (: 352)

Melampyro-Holcetea mollis

Campanulo-Teucrietum scorodoniae KNAPP 1976. In: PASSARGE 1994 b (: 96)

Teucrio-Centaureetum nemoralis MULLER 1962. In: PASSARGE 1994b (: 96)

O Veronico-Hieracietum murorum KLAUCK 1992. In: PASSARGE 1994b typific. superf1. (: 91) [art. 18]

Molinio-Arrhenatheretea

Agrostio-Arrhenatheretum bubosi TELES 1970. In: DÍAZ GONZÁLEZ \& FERNÁNDEZ PRIETO 1994 (: 374$)$

Bromo-Polygonetum bistortae RIVAS-MARTÍNEZ ex MAYOR in MAYOR, DÍAZ, NAVARRo, MARTínez et ANDRÉS 1975. In: Díaz GonzÁlez \& Fernández Prieto 1994 (: 375)

Caro verticillati-Cynosuretum cristati (BELlot et CASASECA) TÜXEN in TÜXEN et OBERDORFER 1958. In: DÍAZ GonzÁlez \& FERNÁNDEZ PRIETO 1994 (: 370)

Centaureo radiatae-Molinietum caeruleae TÜXEN et OBERDORFER 1958. In: DIAZ GONZÁLEZ \& FERNÁNDEZ PRIETO 1994 (: 378)

Lino biennis-Cynosuretum cristati TÚXEN et OBERDORFER 1958. In: DÍAZ GONZÁLEZ \& FERNÁNDEZ PRIETO 1994 (:369)

Malvo moschatae-Arrhenatheretum TÜXEN et OBERDORFER 1958. In: DíAZ GONZÁLEZ \& FERNÁNDEZ PRIETO 1994 (: 372)

Merendero bulbocodii-Cynosuretum cristati TUXXEN et OBERDORFER 1958. In: DÍAZ GONZÁLEZ \& FERNÁNDEZ PRIETO 1994 (: 368)

Plantagini coronopi-Trifolietum fragiferi TÜXEN ex DIAZ 1975. In: DÍAZ GONZÁLEZ \& FERNÁNDEZ PRIETO 1994 (: 383, 384)

Plantagini-Sporoboletum BraUn-BlanQUET 1967. In: Díaz GonzÁlez \& Fernández PRIETo 1994 (: 382 ) Senecioni-Juncetum acutiflori BRAUn-BLANQUET et TÜXEN 1952. In: DíAZ GonZÁLEZ \& FERNÁNDEZ PRIETO 1994 (: 376)

Montio-Cardaminetea

O Dermatocarpion GeISSLER 1976. In: ZECHMEISTER \& MUCINA 1994 typific. superfl. (: 400) [an. 18]

O Mniobryo-Epilobion hornemannii NORDHAGEN 1943. In: ZECHMEISTER \& MUCINA 1994 typific. superfl. (: 400) [art. 20] [sub: Mniobryo-Epilobion hornemannii (NORDHAGEN 1943) ZECHMEISTER et MUCINA 1994]

Mulgedio-Aconitetea

Anemono narcissiflorae-Avenelletum flexuosae KMONÍČEK 1935. In: KLIMENT 1994 (: 64) [neotypus] 
Potametea

$\Delta$ Luronio-Potametum polygonifolii PIETSCH 1986. In: PASSARGE 1994a (: 366)

$\Delta$ Potametum natanti-lucentis UHLIG 1938. In: PASSARGE 1994a (: 345, 346)

Potametum panormito-graminei KoCH 1926. In: PASSARGE 1994a (: 359)

$\triangle$ Potametum pectinato-perfoliati (PASSARGE 1964) DEN HARTOG et SEGAL 1964. In: PASSARGE 1994a (: 347, 348)

$\Delta$ Ranunculo-Elodeetum nuttallii DE LANGE 1972. In: PASSARGE 1994a (: 342,343$)$

$\Delta$ Sparganio-Elodeetum WeBER-OLDECOP 1977. In: PASSARGE 1994a (: 341)

Quercetea ilicis

$\Delta$ Asparago-Juniperetum macrocarpae R. et R. MOLINIER ex BoLòs 1962. In: GÉHU \& BroNDI 1994 (: 141)

Quercetea robori-petraea

Festuco heterophyllae-Quercetum pyrenaicae BRAUN-BLANQUET 1967. In: LOIDI \& HERRERA 1994 (: 169)

Querco-Fagetea

$\triangle$ Aro orientalis-Carpinetum (DOBREscu et Kovács 1973) TÄUBER 1993. In: COLDEA \& CHIFU 1994 (: 319 ) Carpino-Fagetum PaUCA 1941. In: COLDEA et CHIFU 1994 (: 327)

Dentario pentaphylli-Fagetum MaYer et HofManN 1969. In: POLdiNi \& NARdini 1994 (: 241 ) [neotypus]

$\triangle$ Dryopterido carthusianae-Fagetum (BURDUJa, MIHAI et SARBU 1972) CHIFU et ȘTEFAN 1994. In: CHIFU \& ŞTEFAN $1994(: 77,80)$ [neotypus]

$\triangle$ Evonymo nanae-Carpinetum (BoRZa 1937) BoşCalU in SHEGHEdIN, FILIPASCU et BoşCAIU 1977. In: CoLDEA \& CHIFU 1994 (: 319)

$\Delta$ Galio schultesii-Fagetum (BURduja, MrHaI et SARBU 1972) CHIFU et ŞTEFAN 1994. In: ChIFU \& ŞTEFAN 1994 $(: 74,76)$ [neotypus]

O Lathyro hallersteinii-Carpinetum COLDEA 1975. In: COLDEA \& CHIFU 1994 typific. illeg. (: 325) [art. 19]

$\triangle$ Melampyro bihariensis-Carpinetum (BORZA 1941) SOó 1964. In: COLDEA \& CHIFU 1994 (: 312)

O Tilio tomentosae-Carpinetum DonITA 1968 typific. illeg. In: COLDEA \& CHIFU 1994 (: 326 ) [art. 16] Polysticho setiferi-Fraxinetum excelsioris (TÜXEN et OBERDORFER 1958) RIVAS-MARTINEZ ex NAVARRO I982. In: DÍAZ GONZÁLEZ \& FERNÁNDEZ PRIETO 1994 (: 279, 280)

Rosmarinetea

Erinacetalia anthyllidis QUÉZEL 1953. In: DíEZ GARRETAS \& ASENSI MARFIL 1994 (: 544)

O Rosmarinetalia BRAUN-BLANQUET 1931 nom. inval. In: DíEZ GARRETAS \& ASENSI MARFIL 1994 typific. superfl. (: 540)

O Xero-Acanthion QUÉZEL 1953 nom. inval. In: DíEZ GARRETAS \& ASENSI MARFIL 1994 typific. superfl. (: 544) [sub: Xeroacantho-Erinaceion QUÉZEL 1953 em. RIVAS GODAY et RIVAS-MARTíneZ in RIVAS-MARTINEZ 1961) O. BoLòs 1967]

O Xeroacantho-Erinaceion (QUÉZEL 1953) Bolòs 1967 nom. inval. In: DíEZ GaRRETAS \& ASENSI MARFIL 1994 typific. superfl. (: 544)

Lavandulo-Genistion boissieri RIVAS-GODAY \& RIVAS-MARTíneZ 1969. In: DíEZ GARRETAS \& ASENSI. MARFIL 1994 (: 540)

* Convolvulo-Lavanduletum lanatae Rivas Goday et Rivas-MarTínez 1969. In: Díez GarRetas \& ASENSI MARFIL 1994 typific. illeg. (: 540, 541) [art. 19]

Erinaceo anthyllidis-Genistetum longipedis Bolòs et RIGUAL in BOLòs 1967. In: DízZ GARRETAS \& ASENSI MARFIL 1994 (: 544)

Helianthemo-Anthyllidetum argyrophyllae RIVAS GODAY et ESTEVE 1972. In: DíEZ GaRRETAS \& ASENSI MARFL 1994 (: 545)

$\Delta$ Hippocrepido eriocarpae-Pterocephaletum spathulatae (QUÉzEL 1953) RIVAS GODAY et MATÍAS MAYOR 1966. In: DíEZ GARRETAS \& ASENSI MARFIL 1994 (: 545)

Saturejo intricatae-Genistetum boissieri RIVAs GodaY et RIVAs-Martínez 1969 corr. MARTínez PARRAS, PEINADO et Alcaraz 1984. In: Diez GarReTAS \& ASENSI MARFIL. 1994 (: 540)

Saturejo prostratae-Velletum spinosae RIVAS GODAY 1968. In: DíEZ GARRETAS \& ASENSI MARFIL 1994 (: 544$)$ Sideritido osteoxyllae-Teucrietum charideni (Rivas Goday et EsteVe ChUECA 1966) PEInAdo, AlCARAZ, MARTíneZ PARRAS et DE LA CRUZ 1988. In: DíEz GARRETAS \& ASENSI MARFIL 1994 (: 547)

Teucrio-Coridothymetum baeticum RIVAS GODAY et RIVAS-MARTÍNEZ 1969. In: DíEZ GARRETAS \& ASENSI MARFL 1994 (: 542) 
[sub: Teucrio lusitanici-Coridothymetum capitati (RIVAS GODAY et RIVAS-MARTINEZ 1968) RIVAS-MARTíNEZ in DíEZ GARRETAS \& ASENSI MARFIL 1994]

Teucrio lusitanici-Coridothymetum capitati (RIVAS GODAY et RIVAS-MARTíNEZ 1969) RIVAS-MARTINEZ in DIEZ GarReTas et ASENSI MARFIL 1994. In: DíEZ GARRETAS \& ASENSI MARFIL 1994 (: 542)

Ulici baetici-Cistetum clusii RIVAs GODAY et RIVAS-MARTíneZ 1969 corr. AsENSI MARFIL et DíEZ GARRETAS 1994. In: Díez Garretas \& ASEnsi MarflL 1994 (: 542)

Ulici-Halimietum viscosi RIVAS GodaY et RIVAS-MARTínEZ 1968. In: Díez GaRRETAS \& AsENSI MaRFIL 1994 (: 542)

[sub: Ulici baetici-Cistetum clusii RIvas GODAY et RIVAS-MARTínEZ 1968 corr. AsENSI MARFIL et DíEZ GARRETAS 1994]

Sarcornietea

$\Delta$ Sarcocomietum deflexae (BRAUN-BLANQUET 1931) LAMONDÈRE, GéHU et PARADIS 1992. In: GÉHU \& BIONDI 1994 $(: 64,66)$

Stellarietea mediae

Atriplicetum nitentis KNAPP ex SCHREIER 1995. In: WIBKIRCHEN \& KRAUSE 1994 (: 434)

Thlaspietea

Dryopteridetum robertianae KAISER 1926. In: KOLBEK \& SÁDLO 1994 (: 124)

Trifolio-Geranietea

Campanulo bononiensis-Vicietum tenuifoliae. In: DENGLER 1994 (: 270) [neotypus]

Zosteretea

$\Delta$ Zosteretea PignATti 1953. In: GIACCONE et al. 1994 [1995?] (: 136)

* Zosteretalia BÉguinot 1941. In: GiacCONE et al 1994 [1995?] (: 136)

O Zosterion BRAUN-BLANQUET et TÜXEN 1943 nom. inval. In: GiaCCONE et al. 1994 [1995?] typific. superfl. (: 115)

$\Delta$ Cymodoceetum nodosae GIACCONE et PIGNATTl 1967. In: GiacCONE et al. 1994 [1995?] (: 133)

$\triangle$ Posidonietum oceanicae MOLINIER 1958. In: GIACCONE et al. 1994 [1995?] (: 131)

$\Delta$ Zosteretum marinae PignatTI 1953. In: GIACCONE et al. 1994 [1995?] (: 115) [neotypus]

$\Delta$ Zosteretum noltii PIGNATTI 1953. In: GiaCCONE et al. 1994 [1995?] (: 134)

\section{REFERENCES}

BARKMAN J.J., MORAVEC J. \& RAUSChERT S. (1986): Code of phytosociological nomenclature. Vegetatio 67: $145-195$. CHIFU T. \& \$TEFAN N. (1994): Analele Şti., Univ. Al. I. Cuza laşi, Sect. 2 .40: 71-80.

Coldea G. \& ChIFU T. (1994): Phytocoenologia 24: 311-336.

DENGLER J. (1994): Gleditschia 22: 179-321.

Díaz González T.E. \& Fernández Prieto J.A. (1994): Itinera Geobot. 8: 243-528.

Díez Garretas B. \& ASEnSi Marfil A. (1994): Colloq. Phytosoc. 22: 539-553.

Dubyna D.V., NEUHÄUSLOVÁ Z. \& ŠElyAG-SoSONKo J.R. (1995): Preslia 66(1994): 193-216.

Feoli Chiapella L. \& Poldini L. (1994): Stud. Geobot. 13: 3-140.

GÉHU J.-M. \& BIONDI E. (1994): Braun-Blanquetia 13; 1-149.

Giaccone, G., Alongi G., Pizzuto F. \& Cossu A. (1994) [1995?]: Boll. Accad. Gioenia Sci. Nat. Catania 27: $111-157$.

KLIMENT J. (1994): Bull. Slov. Bot. Spoločn. 16: 63-70.

Kolbek J. \& SÁdlo J. (1994): Preslia 66: 115-131.

LOIDI J. \& HERRERA M. (1994): Lazaroa 14: 169-170.

PASSARGE H. (1994a): Phytocoenologia 24: 337-367.

PASSARGE H. (1994b): Tuexenia 14: 83-111.

Poldini L. \& NARdini S. (1994): Stud. Geobot. 13: 215-298.

THEURILLAT J.-P. \& MORAVEC J. (1990): Index of names of syntaxa typified in 1987. Folia Geobot. Phytotax. 26: 101-106. Theurillat J.-P. \& MoraVeC J. (1995): Index of names of syntaxa typified in 1992. Folia Geobot. Phytotax. 30: $363-369$. THEURILLAT J.-P. \& MORAVEC J. (1998): Index of new names of syntaxa published in 1994. Folia Geobot. 33: $441-473$. TOMASELLI M. (1994): Fitosociologia 26: 35-50. 
WIBKIRCHEN R. \& KRAUSE S. (1994): Tuexenia 14: 425-444.

WOLFF P., DIEKJOBST H. \& SCHWARZER A. (1994): Tuexenia 14: 343-380.

ZECHMEISTER H. \& MUCINA L. (1994): J. Veg. Sci. 5: 384-402.

\section{ADDITIONS TO INDEX 1992}

Meliloto-Artemisietea absinthii

Achilleo millefolii-Grindelietum squarrosae KostıLov 1990. In: SOLOMAKHA et al. 1992 (: 197)

Sis y mbrio-Onopordetea

Bromo squarrosi-Sonchetum oleracei KOSTILOV 1990. In: SOLOMAKHA et al. 1992 (: 225)

Chamomillo recutitae-Malvetum mauritianae KostLLV 1990. In: SOLOMAKHA et al. 1992 (: 224)

\section{REFERENCES}

SOLOMAKHA V.A., Kostilov O.V. \& SHELYAG-SOSONKo Y.R. (1992): Synantropna roslinnist Ukrainy. Kyiv. 